\title{
Giant Primary Epithelial Splenic Cyst in an Adolescent Girl - Report of Successful Management of a Rare Case
}

\author{
Kajal $\mathrm{P}^{* 1}$, Rattan $\mathrm{KN}^{1}$, Bhutani $\mathrm{N}^{2}$ and Bhalla $\mathrm{K}^{1}$ \\ ${ }^{1}$ Department of Pediatric Surgery, Pt BDS PGIMS, Rohtak, Haryana, India \\ ${ }^{2}$ Department of Pathology, Pt BDS PGIMS, Rohtak, Haryana, India
}

${ }^{*}$ Corresponding author: Kajal P, Department of Pediatric Surgery, Pt BDS PGIMS, Rohtak, Haryana, India, Tel: +918221000060, E-mail: drpradeepkajal@gmail.com

Citation: Kajal P, Rattan KN, Bhutani N, Bhalla K (2016) Giant Primary Epithelial Splenic Cyst in an Adolescent Girl - Report of Successful Management of a Rare Case. J Surg Oper Care 2(1): 101. doi: 10.15744/2455-7617.2.101

Received Date: June 28, 2016 Accepted Date: July 24, 2016 Published Date: July 25, 2016

\begin{abstract}
Splenic cysts are very unusual in daily surgical practice and less than 1000 cases have been reported in literature till date. These are classified as true or pseudocyst based on presence or absence of epithelial lining. Primary, true or epithelial splenic cysts are even rarer. Most of the cases are asymptomatic until of significant size, at which time they are then detected incidentally on ultrasonography or CT scan. We report a case of 13 years old girl who presented to us with a huge lump in central and left upper part of the abdomen. Ultrasonography and CT scan of the abdomen confirmed the clinical diagnosis of a giant splenic cyst and the patient underwent laparotomy with splenectomy. Histopathological examination of the specimen confirmed it to be a giant epithelial splenic cyst.
\end{abstract}

Keywords: Epithelial cyst; Laparatomy; Pseudocyst; Splenic cyst; Splenectomy

\section{Introduction}

Splenic cysts are very rare lesions in surgical practice. These can be parasitic (hydatid), caused by the parasite Echinococcus granulosus, or nonparasitic [1,2]. Non-parasitic cysts are classified as primary (true, epithelial), lined by an epithelial cover (epidermoid, dermoid, and mesothelial) or endothelial cover (hemangioma, lymphangioma), and secondary (pseudocysts, nonepithelial), which are usually secondary to abdominal trauma [3,4]. Most of the cysts are asymptomatic, and they are incidental findings during abdominal ultrasonography. Laparotomy with splenectomy has been the method of choice for the treatment of primary splenic cysts, but at present the decision about the type of surgical procedure is dictated by the size of the cyst, its relationship to the splenic hilum and amount of normal remnant splenic tissue and whether cyst is symptomatic [5,6]. Recently, we encountered a case of a giant primary splenic cyst which was managed successfully and is being reported here with review of literature.

\section{Case Report}

A 13 years old girl presented to us with complaint of abdominal pain and upper abdominal fullness for previous 4 months. There was no history of fever, vomiting, hematemesis, malaena, jaundice, animal contact or abdominal trauma. There was no history of any immunological disorder either in the patient or the family. On per abdomen examination, there was a large, smooth surfaced, firm lump palpable involving the left hypochondrium, epigastrium, left lumbar region and reaching almost upto the umbilicus (Figure 1). It was moving with respiration and the upper limit was not reachable. Rest of the abdomen was normal. Digital rectal examination was also normal. All routine hematological and biochemical investigations were within normal range. Hematological tests for human immunodeficiency virus (HIV), hepatitis C virus (HCV) and hepatitis B virus (HBV) viz. ELISA for HIV, anti-HCV and HBsAg were negative. X-ray chest revealed slight elevation of left diaphragm. Abdominal ultrasonography revealed a giant splenic unilocular cystic lesion. Casoni's skin test and complement fixation test for Echinococcus granulosus were negative. Stool examination done over 3 consecutive days was found to be normal. Computed tomography confirmed the splenic localization of the noncalcified single large cyst with dimensions of $22 \times 17 \times 15 \mathrm{~cm}$ with thin, compressed rim of normal parenchyma posterolaterally (Figure 2). At laparotomy, a huge splenic cyst of more than $20 \mathrm{~cm}$ of maximum diameter was found with almost total displacement and compression of remaining splenic parenchyma (Figure 3 ). However due to cyst size and location, preservation of spleen was considered impossible and total splenectomy was carried out after decompression of the cyst by aspiration of about $2000 \mathrm{ml}$ of turbid serous fluid from it (Figure 4). The post-operative period was uneventful and patient was discharged in fair health on 5th post-operative day. The histopathological examination of excised specimen revealed the cyst to be lined by tubulocolumnar, pseudostratified (mesothelial line) epithelium. Focally, the lining epithelium was flattened and 
the cyst wall was composed of fibrocollagenous tissue (Figure 5). The cyst was filled with eosinophillic material and foamy and hemosiderin-laden macrophages. So, the histopathological diagnosis was primary epithelial splenic cyst. Aspirated cystic fluid showed no evidence of malignancy. Patient had received Hemophilus influenza, Pneumococcal (unconjugated 23 valent) and meningococcal vaccine 2 weeks before surgery and antibiotic prophylaxis was given after surgery for a period of 6 months. The patient is on regular follow up for last one year and doing very well.

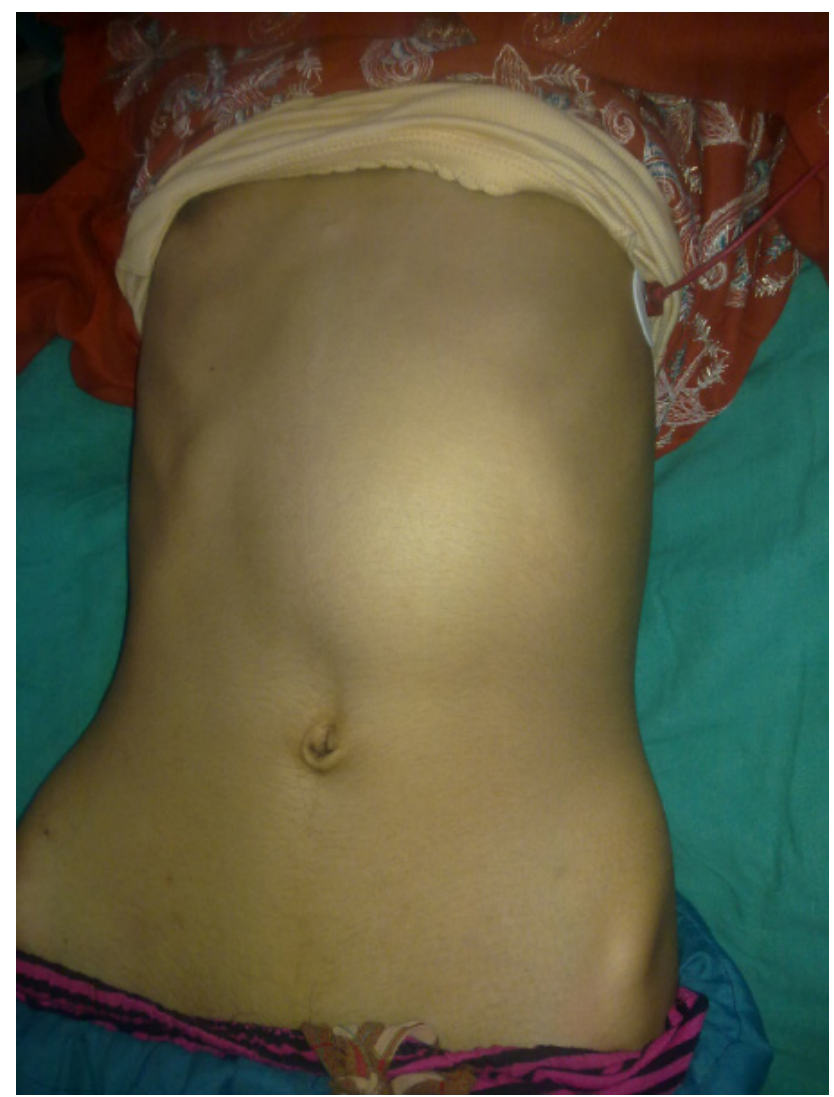

Figure 1: Clinical photograph showing a huge lump involving the upper half of the abdomen

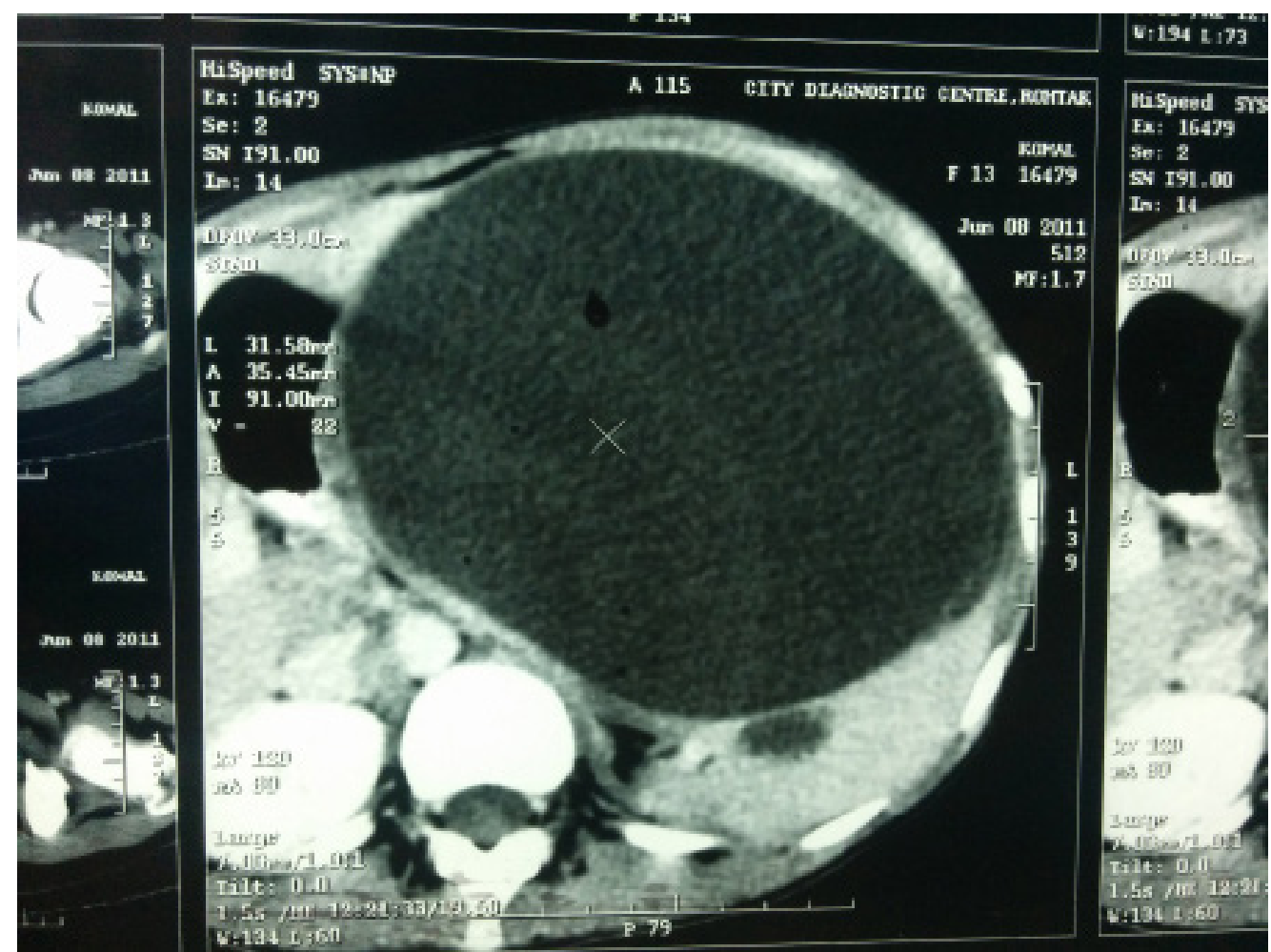

Figure 2: Computed tomographic image of the upper abdomen demonstrating a very large, homogeneous splenic cyst compressing the normal splenic parenchyma and adjacent viscera and even crossing the midline 


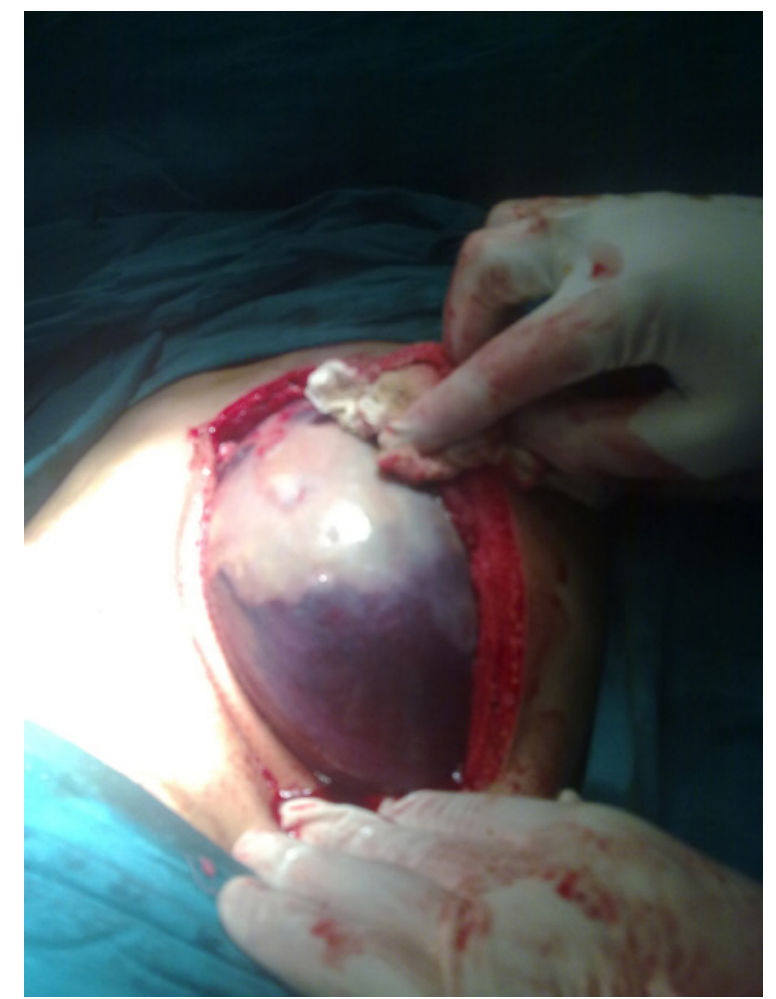

Figure 3: Intraoperative image of splenic cyst after separation of adhesions

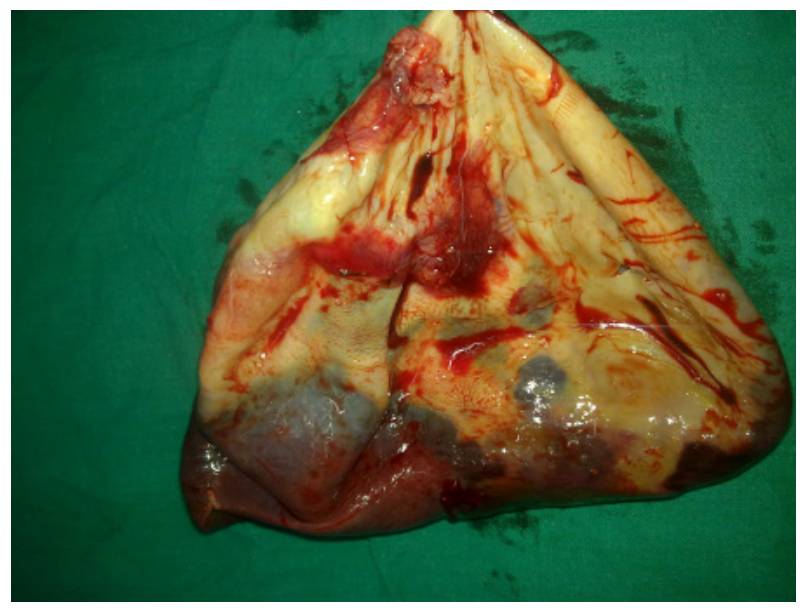

Figure 4: Resected specimen of spleen with giant cyst after decompression

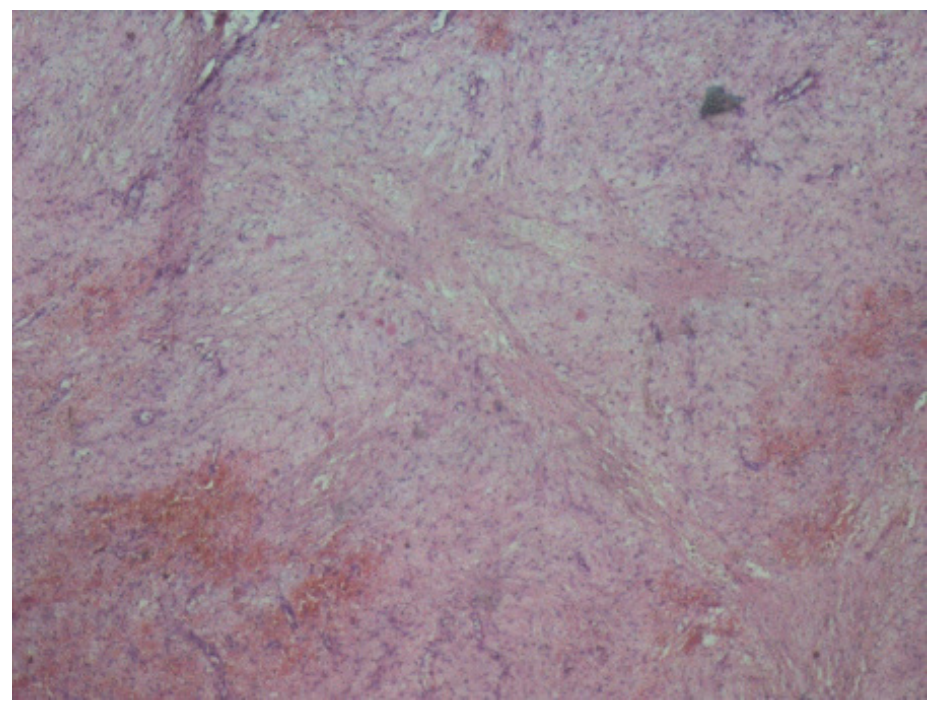




\section{Discussion}

Splenic cysts have been classified based on the presence or absence of an epithelial lining, etiology, pathogenesis, etc. Martin classified splenic cysts as type 1 cysts, which are true cysts having lining epithelium, and type 2 cysts, which are false cysts without lining epithelium [7]. Pseudocysts are usually post-traumatic, due to failure of organization of hematomas located beneath the capsule or in the splenic parenchyma, and rarely they may occur in splenic abscess or splenic infarction [8]. Depending on the causative agent splenic cysts can be divided into two types: parasitic cysts and non-parasitic cysts. Parasitic cysts are usually seen in endemic areas and are caused mainly by Echinococcus granulosus infestation. A new classification based on the true pathogenesis of cyst divides non-parasitic splenic cysts as congenital, neoplastic, traumatic, and degenerative [9]. Primary splenic cysts constitute $10 \%$ of all nonparasitic cysts of the spleen. Primary epithelial splenic cyst is a rare condition with an incidence rate of $0.07 \%$ as reported in a review of 42327 autopsies [10].

The pathogenesis of primary splenic cysts is not clear. The various hypotheses which have been proposed are -

\section{Mesothelial invagination theory}

In case of congenital cysts, it is postulated that during development there is invasion of mesothelial lining along with the capsule. As the lining has pluripotent nature, it has propensity to undergo metaplasia and secretion of fluid, leading to the formation of cysts [11].

\section{Lymph space theory}

According to this theory the cysts may arise from the normal lymph spaces in the spleen.

\section{Endodermal inclusion theory}

Endodermal inclusion theory proposes that epithelial splenic cysts develop by true metaplasia of a heterotopic endodermal inclusion within the spleen.

Splenic cysts are predominantly seen in the second and third decades; however, they can be seen in paediatric age group; more common in females as compared to males. Usually small cysts are symptomless. A painless mass in the left hypochondrium is the main presentation in 30\%-40\% cases. There may be localized pain or referred pain due to mass effect. Occasionally the patients may present with thrombocytopenia. The initial symptoms are mainly due to pressure effect, including nausea and belching pain in the abdomen. Pleuritic pain and persistent cough are also the presenting features. Occasionally they present with complications like infection, rupture and haemorrhage. Even presence of potentially pathogenic and invasive Blastocystis sp. which usually are opportunistic emerging pathogens in immunocompromised individuals, has been reported in splenic cyst fluid [12].

Physical examination often reveals a left hypochondriac mass. The routine haematological and biochemical investigations are usually within normal limits. Most of primary cysts are clinically silent and are diagnosed incidentally on USG.

Whenever there is a lump in the left hypochondriac region, the clinician should exclude other causes of splenomegaly, i.e. infectious mononucleosis, fever of unknown origin, haemolytic anaemia, chronic leukemias, collagen vascular disease, and liver diseases. Serological markers play key role in such circumstances. USG is a good non-invasive tool for screening and confirming the cystic nature of a mass [2]. Characteristically, on USG the cyst appears as an anechoic mass with thin walls and septations or irregular walls. CT and magnetic resonance imaging (MRI) may give guidelines, with regard to the morphology of cyst, the nature of fluid, the exact location and its relationship with adjacent structures. However, definite diagnosis is possible only after splenectomy when epithelial lining is confirmed by histopathology along with immunohistochemistry.

Primary epithelial cysts are usually solitary, but can be multiple. Histologically, primary splenic cysts have epithelial lining, i.e., flattened, low cuboidal, low columnar or squamous type and unilayered or multilayered with benign nuclear features [8].

The differential diagnoses for a cyst in the spleen include parasitic Echinococcal disease, congenital cyst, post-traumatic pseudocyst, infarction, infection, pyogenic splenic abscess, pancreatic pseudocyst, metastatic disease, and cystic neoplasms like haemangioma/ lymphangioma (diffuse lymphangiomatosis of spleen).

Previously, splenectomy was the treatment of choice for splenic cysts. Recently, the approach has changed towards conservative surgery in view of overwhelming sepsis after splenectomy leading to raised mortality. The splenic cysts with a diameter more than $4 \mathrm{~cm}$ should be treated surgically [13]. Surgery is primarily recommended for the prevention or treatment of complications. Decisions about the exact surgical procedure are based on the size of the cyst, its relation to the splenic hilar vessels and parenchyma, and the amount of healthy splenic tissue remaining [14]. Today, the options of management are percutaneous aspiration or percutaneous drainage, partial splenectomy with a stapler or harmonic scalpel, total cystectomy, marsupialization or cyst decapsulation (unroofing), laparoscopic puncture and creation of a cyst peritoneal window [15-17]. Access can be either by open laparotomy or laparoscopy. Partial splenectomy preserves more than $25 \%$ of the splenic parenchyma, which is the minimal splenic tissue to preserve immunologic protection without increasing the risk of recurrence. Although the literature offers the possibility of providing conservative or semi conservative treatment, in our case, the cyst was huge which reduced the spleen to a 
small remnant, for which reason it would have been impossible to perform a simple cystectomy or partial splenectomy to preserve the spleen, and it was encompassing the hilum of the spleen. Hence, total splenectomy, vaccination, and long-term antibiotic prophylaxis is a viable alternative when it is needed as in our case.

Any type of conservative surgical treatment modality has little value in cases such as a very large cyst in the hilum of the spleen, an intrasplenic cyst almost surrounded by splenic parenchyma, with dense vascular adhesions to surrounding structures, and multiple cysts. In such circumstances, splenectomy either by open approach or by laparoscopic approach is the treatment of choice.

\section{Conclusion}

True giant primary splenic cysts are rare. Clinical presentation varies from asymptomatic to left hypochondriac pain and lump abdomen. The factors which govern the decision about the type of surgical procedure are - the size of the cyst, its relationship to the splenic hilum and amount of normal remnant splenic tissue. If the cyst is very large and almost completely covered by splenic parenchyma, or if it is located in the splenic hilum, complete splenectomy is recommended, because of the risk of intractable bleeding from the spleen. Conservative and minimal surgical approach should be employed for smaller sized and peripherally located splenic cysts.

\section{References}

1. Avital S, Kashtan H (2003) A large epithelial splenic cyst. N Engl J Med 349: 2173-4.

2. Safioleas M, Misiakos E, Manti C (1997) Surgical treatment for splenic hydatidosis. World J Surg 21: 374-8.

3. Reddi VR, Reddy MK, Srinivas B, Sekhar CC, Ramesh O (1998) Mesothelial splenic cyst- A case report. Ann Acad Med Singapore 27: 880-2.

4. Heidenreich A, Canero A, di Pasquo A (1996) Laparoscopic approach for treatment of a primary splenic cyst. Surg Laparosc Endosc 6: 243-6.

5. Hansen MB, Moller AC (2004) Splenic cysts. Surg Laparosc Endosc percutan Tech 14: 316-22.

6. Smith ST, Scott DJ, Burdick JS, Rege RV, Jones DB (2001) Laparoscopic marsupialization and hemisplenectomy for splenic cysts. J Laparoendosc Adv Surg Tech A 11:243-9.

7. Martin JW (1958) Congenital splenic cysts. Am J Surg 96: 302-8.

8. Ingle SB, Hinge CR, Jatal SN (2013) An interesting case of primary epithelial cyst of spleen. Indian J Pathol Microbiol 56: 181-2.

9. Morgenstern L (2002) Nonparasitic splenic cysts: pathogenesis, classification, and treatment. J Am Coll Surg 194: $306-14$.

10. Macheras A, Misiakos EP, Liakakos T, Mpistarakis D, Fotiadis C, et al. (2005) Non-parasitic splenic cysts: a report of three cases. World J Gastroenterol 11: 6884-7.

11. Belekar D, Desai A, Dewoolkar A, Dewoolkar V, Bhutala U (2010) Splenic epithelial cyst: A rare entity. Int J Surg 22: 1-18.

12. Santos HL, Sodré FC, de Macedo HW (2014) Blastocystis sp. in splenic cysts: causative agent or accidental association? A unique case report. Parasit Vectors 7: 207. doi: 10.1186/1756-3305-7-207.

13. Szczepanik AB, Meissner AJ (2009) Partial splenectomy in the management of nonparasitic splenic cysts. World J Surg 33: 852-6.

14. Kalinova K (2005) Giant pseudocyst of the spleen: A case report and review of the literature. J Indian Assoc Pediatr Surg 10: 176-8.

15. López Cano A, Muñoz Benvenuty A, Méndez Pérez C, Herrera M, Ortiz Acero I, et al. (2001) Treatment of non-parasitic splenic cyst with percutaneous injection of alcohol. Gastroenterol Hepatol 24: 199-201.

16. Matsutani T, Uchida E, Yokoyama T, Matsushita A, Matsuda A, et al. (2009) Laparoscopic Unroofing of a Large Pseudocyst of the Spleen: Report of a Case. J Nippon Med Sch 76: 319-22.

17. Daga G, Mittal V, Singh RJ, Sood N (2011) Epithelial cyst of the spleen. J Indian Assoc Pediatr Surg 16: 18-20.

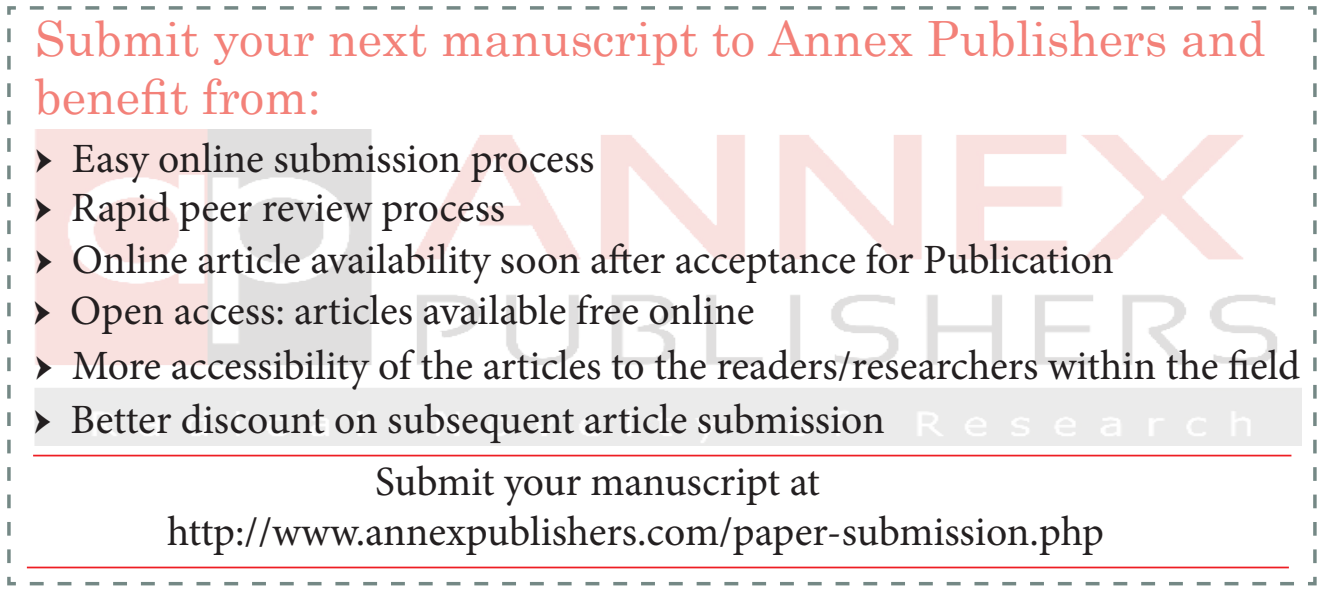

